\title{
Oral Sulfate Solution for Precolonoscopy Bowel Preparation: A New Kid on the Block
}

\author{
Ashish Kumar Jha ${ }^{1}$ \\ ${ }^{1}$ Department of Gastroenterology, Indira Gandhi Institute of \\ Medical Sciences, Patna, Bihar, India
}

J Digest Endosc 2019;10:178-179

Colonoscopy is a widely used procedure which requires prior cleansing of the bowel. Optimal bowel cleansing is essential for a successful and accurate examination of the bowel. Inadequate bowel cleansing during colonoscopy can result in missed lesions, difficult and incomplete procedures, prolonged cecal intubation time, and increased risk of procedural complications. The adequacy of bowel cleansing mainly depends on the type of cleansing agents, volume of preparation, mode of administration (single dose vs. split dose, single day vs. two days), use of adjunct agents, the preparation-to-colonoscopy interval, and associated comorbidities in patients. ${ }^{1,2}$ Other factors for adequate bowel cleansing may include low residue diet, liberal fluid intake, and proper bowel preparation instructions.

Many different bowel cleansing preparations have been developed, all of which have specific pros and cons. Data regarding the comparison of various colonoscopy preparation regimens are still variable. Polyethylene glycol-electrolyte solution (PEG-ELS)-based solutions are most commonly used precolonoscopy cleansing agent because of an excellent safety profile. A split-dose regimen of 4-L PEG-ELS is recommended by the American College of Gastroenterology as an optimal choice for colonoscopy. Studies showed that 4-L split-dose PEG-ELS is better than other bowel preparation agents for colonoscopy with comparable compliance, favorable overall experience, and willingness to repeat the same preparation. However, approximately 5 to $15 \%$ of the patients poorly tolerate PEG-ELS, mostly due to large-volume PEG-ELS ingestion. ${ }^{3}$ Volume-related adverse effects of preparation regimens can be minimized with the use of low-volume preparations, split-dose regimen, or combination regimens. Two-liter split-dose PEG-ELS preparation, sulfate-based preparations, and specially formulated preparations such as Clensia, NER1006, and Prepopik are currently available low-volume bowel cleansing regimens for colonoscopy. ${ }^{4}$

Low-volume split-dose regimens address volume-related adverse event profile and are associated with better bowel preparation and higher patient satisfaction than with

\footnotetext{
Address for correspondence

Ashish Kumar Jha, MD, DM, Department of Gastroenterology, Indira Gandhi Institute of Medical Sciences, Sheikhpura, Bailey Road, Patna 800014, Bihar, India (e-mail: ashishjhabn@yahoo.co.in).
}

single-dose regimens. Introduction of oral phosphate and sulfate salts offered the advantage of low intake volume, low risk of gastrointestinal irritation, and less volume-related adverse events compared with other available options. Oral phosphate salts have been associated with acute phosphate nephropathy, and rarely chronic renal damage. Oral sulfate salts (OSSs) have recently been approved for precolonoscopy bowel cleansing. Because sulfate absorption from the intestinal tract is saturable, serum sulfate concentrations increase only minimally after ingestion. Excretion of OSS in the kidney is not accompanied by calcium excretion and acute phosphate nephropathy. Use of OSS has not been associated with clinical manifestations of kidney injury but clinically nonsignificant serum electrolyte disturbances. ${ }^{5}$

Studies have shown that OSS is a safe and effective bowel cleansing agent for colonoscopy. The efficacy of OSS in cleansing the colon ranges from 82 to $98 \%{ }^{6-9}$ Studies have shown better efficacy and comparable adverse events profile of split-dose OSS regimen compared with split-dose PEG solution regardless of dose. In a study, OSS subjects reported slightly increased gastrointestinal events (abdominal distension, pain, nausea, vomiting, or abdominal discomfort) $(p=0.009)$ in the single-day preparation but not in the split-dose OSS regimen. ${ }^{7}$ Colonoscopy preparation using split-dose low-volume OSS appears to be cost-effective compared with PEG-ELS with a cost saving of $\$ 16.01$ per patient per year for the OSS cohort. ${ }^{10}$ It is worth mentioning that data are available regarding the usefulness of OSS as a bowel cleansing agent for colonoscopy; however, most of the studies were performed on outdoor patients. Data regarding the efficacy and safety of OSS in the elderly patients with or without comorbidities and hospitalized patients are very limited.

In the current issue, "Oral sulfate solution versus polyethylene glycol as a single day preparation for colonoscopy: a randomized control trial" by Shah et $\mathrm{al}^{11}$ showed superior efficacy and comparable adverse events profile of OSS compared with PEG regimen for bowel preparation for colonoscopy. However, the adequate bowel cleansing was achieved

Copyright $\odot 2019$ Society of

Gastrointestinal Endoscopy of India

\section{License terms}

$10.1055 / \mathrm{s}-0039-3401912$

ISSN 0976-5042. 
in only half of the patients in the PEG group and two-thirds of patients in the OSS group. Major limitations of the current study are lower rate of adequate bowel preparation in both arms of the study, and inclusion of outdoor patients only.

In conclusion, low-volume preparation regimens are gaining mainstream acceptance for precolonoscopy bowel cleansing. OSS appears to be a safe and effective preparation agent for colonoscopy in outdoor patients, but current data does not support use of OSS as a bowel cleansing agent for colonoscopy in hospitalized patients with comorbidities. Further studies are required to recommend OSS as the preferred bowel preparation agent for colonoscopy.

\section{Conflict of Interest}

None declared.

\section{References}

1 Johnson DA, Barkun AN, Cohen LB, et al. US Multi-Society Task Force on Colorectal Cancer. Optimizing adequacy of bowel cleansing for colonoscopy: recommendations from the US multi-society task force on colorectal cancer. Gastroenterology 2014;147(4):903-924

2 Jha AK, Chaudhary M, Jha P, et al. Polyethylene glycol plus bisacodyl: a safe, cheap, and effective regimen for colonoscopy in the South Asian patients. JGH Open 2018;2(6):249-254

3 Enestvedt BK, Tofani C, Laine LA, Tierney A, Fennerty MB. 4-Liter split-dose polyethylene glycol is superior to other bowel preparations, based on systematic review and meta-analysis. Clin Gastroenterol Hepatol 2012;10(11):1225-1231
4 Jha AK. How effective are low-volume solutions for oral colonoscopy bowel preparation? J Dig Endosc 2019;10:6-8

5 Moulin B, Ponchon T. A comparative review of use of sulphate and phosphate salts for colonoscopy preparations and their potential for nephrotoxicity. Endosc Int Open 2018;6(10):E1206-E1213

6 Rex DK, Di Palma JA, Rodriguez R, McGowan J, Cleveland M. A randomized clinical study comparing reduced-volume oral sulfate solution with standard 4-liter sulfate-free electrolyte lavage solution as preparation for colonoscopy. Gastrointest Endosc 2010;72(2):328-336

7 Di Palma JA, Rodriguez R, McGowan J, Cleveland MV. A randomized clinical study evaluating the safety and efficacy of a new, reduced-volume, oral sulfate colon-cleansing preparation for colonoscopy. Am J Gastroenterol 2009;104(9):2275-2284

8 Yang HJ, Park SK, Kim JH, et al. Randomized trial comparing oral sulfate solution with 4-L polyethylene glycol administered in a split dose as preparation for colonoscopy. J Gastroenterol Hepatol 2017;32(1):12-18

9 Kwak MS, Cha JM, Yang HJ, et al. Safety and efficacy of low-volume preparation in the elderly: oral sulfate solution on the day before and split-dose regimens (SEE SAFE) study. Gut Liver 2019;13(2):176-182

10 Huynh L, Yermakov S, Davis M, et al. Cost-analysis model of colonoscopy preparation using split-dose reduced-volume oral sulfate solution (OSS) and polyethylene glycol with electrolytes solution (PEG-ELS). J Med Econ 2016;19(4):356-363

11 Shah B, et al. Oral sulphate solution versus polyethylene glycol as a single day preparation for colonoscopy: a randomized control trial. J Dig Endosc; 2019, this issue 